\title{
Refined theory for linearly elastic plates: Laminae and laminates
}

\author{
David J. Steigmann \\ Department of Mechanical Engineering \\ University of California \\ Berkeley, CA. 94720 \\ USA
}

Abstract: It is well known that bending and stretching modes of deformation in linearly-elastic plates decouple at leading order in thickness provided that the mid-plane is a plane of reflection symmetry of the three-dimensional material properties. This situation being the exception rather than the norm, it is important to have a model that accommodates coupling in a manner consistent with the threedimensional nature of the problem while retaining the analytical tractability of conventional engineering plate theory. Here, this is achieved through an expansion of the potential energy of the plate in powers of its thickness to derive an optimal approximate two-dimensional model for equilibria. The variables are the displacement field of a surface parallel to the lateral surfaces of the plate and associated director fields that emerge naturally in the expansion procedure. To achieve a model that is as accurate as possible, the directors are constrained in accordance with necessary conditions arising in the exact three-dimensional theory. These are incorporated at the level of the energy functional, yielding a model accurate to third order in the small thickness.

Keywords: plate theory, linear elasticity

\section{Introduction}

Current research on the relationship between three-dimensional elasticity and two-dimensional models of plates and shells is based either on the method of gamma convergence [1], concerned with the limiting variational problem as thickness tends to zero, or on asymptotic expansions of the weak forms of the three-dimensional equilibrium equations in powers of thickness [2]. While these techniques have yielded rigorous derivations of membrane theory and pure-bending theory, neither has yielded a model of the classical type in which membrane and bending effects are accommodated simultaneously in a single model. An important exception occurs when the effects of stretching and bending decouple at leading order; i.e., when the plate midsurface is a plane of reflection symmetry of the material properties and the deformation is sufficiently small. In this circumstance the method of gamma convergence may be applied [3] and delivers a rigorous leading-order model for stretching and bending deformations. Our concern here is with the more general case in which such symmetry need not obtain. In this case we use the method discussed in [4-6], which delivers precisely the same model as that obtained via the method of gamma convergence in circumstances when the latter may be applied [5]. However, our method, while far simpler in concept, is not limited to the case of mid-plane reflection symmetry. It is 
similar in some respects to the method Koiter [7-10] used to establish a highly successful small-strain model of linearly elastic isotropic shells. His is not a limit model in the sense of gamma convergence or asymptotic analysis. Nevertheless, it has been justified through careful asymptotic estimates of solutions to the three-dimensional theory for a thin body [2]. We have used this method previously [4-6] to generate models for linearly elastic isotropic or transversely isotropic plates having reflection symmetry with respect to the mid-plane. Here we extend the idea to plates with arbitrary symmetry, including laminates.

Standard notation is used throughout. We use bold face for vectors and tensors and indices to denote their components. Latin indices take values in $\{1,2,3\}$; Greek in $\{1,2\}$. The latter are associated with surface coordinates and attendant vector and tensor components. A dot between bold symbols is used to denote the standard inner product. Thus, if $\mathbf{A}_{1}$ and $\mathbf{A}_{2}$ are second-order tensors, then $\mathbf{A}_{1} \cdot \mathbf{A}_{2}=\operatorname{tr}\left(\mathbf{A}_{1} \mathbf{A}_{2}^{t}\right)$, where $\operatorname{tr}(\cdot)$ is the trace and the superscript ${ }^{t}$ is used to denote the transpose. The norm of a tensor $\mathbf{A}$ is $|\mathbf{A}|=\sqrt{\mathbf{A} \cdot \mathbf{A}}$. The linear operators $\operatorname{Sym}(\cdot)$ and $S k w(\cdot)$ deliver the symmetric and skew parts of their second-order tensor arguments, and the same symbols are used to denote the linear spaces of symmetric and skew tensors. The notation $\otimes$ identifies the standard tensor product of vectors. If $\mathbf{C}$ is a fourth-order tensor, then $\mathbf{C}[\mathbf{A}]$ is the second-order tensor with orthogonal components $C_{i j k l} A_{k l}$. Finally, we use symbols such as Div and $D$ to denote the three-dimensional divergence and gradient operators, while div and $\nabla$ are reserved for their two-dimensional counterparts. Thus, for example, $\operatorname{Div} \mathbf{A}=A_{i j, j} \mathbf{e}_{i}$ and $\operatorname{div} \mathbf{A}=A_{i \alpha, \alpha} \mathbf{e}_{i}$, where $\left\{\mathbf{e}_{i}\right\}$ is an orthonormal basis and subscripts preceded by commas are used to denote partial derivatives with respect to Cartesian coordinates.

The three-dimensional equation of equilibrium without body force is

$$
\operatorname{Div} \tilde{\mathbf{P}}=\mathbf{0}
$$

where

$$
\tilde{\mathbf{P}}=\mathbf{C}[\tilde{\mathbf{H}}] .
$$

is the linear approximation to the Piola stress in the absence of residual stress, $\tilde{\mathbf{H}}=D \tilde{\mathbf{u}}$ is the displacement gradient, $\tilde{\mathbf{u}}(\mathbf{x})$ is the three-dimensional displacement field defined on a material region $R$ and $\mathbf{C}$ is the fourth-order tensor of elastic moduli. The moduli possess the usual minor and major symmetries, the latter ensuring that

$$
\tilde{\mathbf{P}}=U_{\tilde{\mathbf{H}}},
$$

where

$$
U(\tilde{\mathbf{H}})=\frac{1}{2} \tilde{\mathbf{H}} \cdot \mathbf{C}[\tilde{\mathbf{H}}] .
$$

The laminae are assumed to have uniform material properties, so that $\mathbf{C}$ is constant in each lamina. Further, we impose the usual requirement that the strain-energy function be positive definite on the linear space of symmetric tensors:

$$
\mathbf{A} \cdot \mathbf{C}[\mathbf{A}]>0 \text { for all non-zero } \mathbf{A} \in \text { Sym, }
$$

and the minor symmetries of $\mathbf{C}$ yield $\mathbf{A} \cdot \mathbf{C}[\mathbf{A}]=0$ if and only if $\mathbf{A} \in S k w$. This in turn yields the 
strong-ellipticity condition

$$
\mathbf{v} \otimes \mathbf{w} \cdot \mathbf{C}[\mathbf{v} \otimes \mathbf{w}]>0 \quad \text { for all } \quad \mathbf{v} \otimes \mathbf{w} \neq \mathbf{0}
$$

In particular, $\mathbf{v} \otimes \mathbf{w}=\mathbf{0}$ if and only if $\operatorname{Sym}(\mathbf{v} \otimes \mathbf{w})$ vanishes.

Our approach is based on a development of the potential energy

$$
\mathcal{E}[\tilde{\mathbf{u}}]=\int_{R} U d v-\int_{\partial R_{t}} \tilde{\mathbf{t}} \cdot \tilde{\mathbf{u}} d a
$$

of the three-dimensional plate in powers of its thickness, where

$$
\tilde{\mathbf{t}}=\tilde{\mathbf{P}} \mathbf{n}
$$

is assigned as a dead traction on a part $\partial R_{t}$ of the boundary $\partial R$ with exterior unit normal $\mathbf{n}$. Here $\partial R_{t}=\partial R \backslash \partial R_{u}$, where $\partial R_{u}$ is the part where displacement is prescribed. It is, of course, well known that (1) and (8) are the Euler equation and natural boundary condition associated with $\mathcal{E}$.

We seek the optimal expression for the functional $E[\mathbf{u}]$ in the expansion

$$
\mathcal{E}=E+o\left(h^{3}\right)
$$

where $h$ is the plate thickness and $\mathbf{u}$ is the displacement field of a surface $\Omega$ parallel to the lateral surfaces of the plate; this is the mid-plane in the case of laminae, and the interfacial plane of the laminae in the case of a two-ply laminate. The associated Euler equations furnish an approximate system for the analysis of equilibria of the thin plate.

The desired expression, given in Sections 2 and 3, is of the form

$$
E[\mathbf{u}]=\int_{\Omega}[F(\nabla \mathbf{u}, \nabla \nabla \mathbf{u})-\mathbf{g} \cdot \mathbf{u}] d a-\int_{\partial \Omega_{t}}\left(\mathbf{f} \cdot \mathbf{u}+\mathbf{c} \cdot \mathbf{u}_{\nu}\right) d s,
$$

where $\mathbf{u}(\mathbf{r})$ is the displacement of the plane $\Omega$ on which points have (two-dimensional) position $\mathbf{r} ; \nabla(\cdot)$ is the gradient with respect to $\mathbf{r} ; \nabla \nabla(\cdot)$ is the second gradient; $\mathbf{f}, \mathbf{c}$ and $\mathbf{g}$, respectively, are the assigned force and couple per unit length of a part $\partial \Omega_{t}$ of the boundary curve $\partial \Omega$ and the assigned distributed load per unit area of $\Omega ; \mathbf{u}_{\nu}=(\nabla \mathbf{u}) \boldsymbol{\nu}$ is the derivative of the displacement in the direction of the in-plane exterior unit normal $\boldsymbol{\nu}$ to the boundary; and $F$ is a suitable areal strain-energy density. We assume $\mathbf{u}$ and $\mathbf{u}_{\nu}$ to be specified on $\partial \Omega_{u}=\partial \Omega \backslash \partial \Omega_{t}$.

The development of the Euler equations and natural boundary conditions is outlined in [4]. In Cartesian tensor notation, these are

$$
T_{i \alpha, \alpha}+g_{i}=0 \quad \text { in } \Omega
$$

and

$$
T_{i \alpha} \nu_{\alpha}-\left(M_{i \alpha \beta} \nu_{\alpha} \tau_{\beta}\right)_{s}=f_{i}, \quad M_{i \alpha \beta} \nu_{\alpha} \nu_{\beta}=c_{i} \quad \text { on } \quad \partial \Omega_{t},
$$

respectively, where

$$
T_{i \alpha}=N_{i \alpha}-M_{i \beta \alpha, \beta}, \quad \text { with } \quad N_{i \alpha}=\partial F / \partial u_{i, \alpha} \quad \text { and } \quad M_{i \alpha \beta}=\partial F / \partial u_{i, \alpha \beta}
$$

Here $u_{i}=\mathbf{u} \cdot \mathbf{e}_{i}$ are the orthogonal components of $\mathbf{u}$, the subscript ${ }_{s}$ represents the arclength derivative along $\partial \Omega$ (traversed counterclockwise), $\boldsymbol{\tau}=\mathbf{k} \times \boldsymbol{\nu}$ is the unit tangent to $\partial \Omega$ and subscripts preceded 
by commas are used to denote partial derivatives with respect to the in-plane Cartesian coordinates $r_{\alpha}=\mathbf{r} \cdot \mathbf{e}_{\alpha}$.

\section{Laminae}

\subsection{Kinematics and energetics}

The reference placement of the plate is described by

$$
\mathbf{x}=\mathbf{r}+\varsigma \mathbf{k}
$$

where $\mathbf{r} \in \Omega$ (the midplane), $\mathbf{k}$ is the fixed orientation of the plate and $\varsigma \in[-h / 2, h / 2]$. Henceforth we regard $h$ as being small in the sense that $h / l \ll 1$, where $l$ is any other length scale such as a spanwise dimension. It simplifies matters to adopt $l$ as the unit of length (i.e.; $l=1, h \ll 1$ ).

The origin of the position $\mathbf{r}$ is assumed to lie on $\Omega$. Let

$$
\hat{\mathbf{u}}(\mathbf{r}, \varsigma)=\tilde{\mathbf{u}}(\mathbf{r}+\varsigma \mathbf{k}) \quad \text { and } \quad \hat{\mathbf{H}}(\mathbf{r}, \varsigma)=\tilde{\mathbf{H}}(\mathbf{r}+\varsigma \mathbf{k})
$$

and let $\nabla(\cdot)$ and $(\cdot)^{\prime}$, respectively, stand for the (two-dimensional) gradient with respect to $\mathbf{r}$ at fixed $\varsigma$ and the derivative $\partial(\cdot) / \partial \varsigma$ at fixed r. Further, let

$$
\mathbf{1}=\mathbf{I}-\mathbf{k} \otimes \mathbf{k}
$$

where $\mathbf{I}$ is the identity for three-space; this is the projection onto the translation (vector) space $\Omega^{\prime}$ of $\Omega$. In [4] this is used to derive

$$
\tilde{\mathbf{H}} \mathbf{1}=\nabla \hat{\mathbf{u}}, \quad \tilde{\mathbf{H}} \mathbf{k}=\hat{\mathbf{u}}^{\prime}
$$

yielding the orthogonal decomposition

$$
\hat{\mathbf{H}}=\nabla \hat{\mathbf{u}}+\hat{\mathbf{u}}^{\prime} \otimes \mathbf{k}
$$

The subsequent development requires

$$
\mathbf{H}_{0}=\nabla \mathbf{u}+\mathbf{a} \otimes \mathbf{k}, \quad \mathbf{H}_{0}^{\prime}=\nabla \mathbf{a}+\mathbf{b} \otimes \mathbf{k} \quad \text { and } \quad \mathbf{H}_{0}^{\prime \prime}=\nabla \mathbf{b}+\mathbf{c} \otimes \mathbf{k},
$$

where $\mathbf{H}_{0}^{(n)}=\hat{\mathbf{H}}_{\mid \varsigma=0}^{(n)}$ are obtained by differentiating (18) with respect to $\varsigma$, and

$$
\mathbf{u}=\hat{\mathbf{u}}_{0}, \quad \mathbf{a}=\hat{\mathbf{u}}_{0}^{\prime}, \quad \mathbf{b}=\hat{\mathbf{u}}_{0}^{\prime \prime} \quad \text { and } \quad \mathbf{c}=\hat{\mathbf{u}}_{0}^{\prime \prime \prime}
$$

are mutually independent functions of $\mathbf{r}$. These are the coefficient vectors in the displacement ansatz

$$
\hat{\mathbf{u}}=\mathbf{u}+\varsigma \mathbf{a}+\frac{1}{2} \varsigma^{2} \mathbf{b}+\frac{1}{6} \varsigma^{3} \mathbf{c}+\ldots
$$

It is known that in the presence of strong ellipticity, and for standard mixed boundary-value problems, displacement fields satisfying (1) and (2) are of class $C^{\infty}$ in the interior of the body [11]. Accordingly, the displacement ansatz is valid without qualification and may be extended as necessary to include higher-order terms. Because of this the degree of regularity implied by (21) may be imposed without loss of generality in the course of constructing a potential energy functional for the plate. 
Later, we make use of the fact that states of plane stress minimize the three-dimensional strainenergy function pointwise. Accordingly, such states are energetically optimal provided that they are compatible with the boundary data. Indeed, in plate theory it is customary to generate approximate expressions for boundary data that are consistent with restrictions imposed on the deformation in the interior. Thus, we follow the long-standing practice of subordinating boundary conditions to other aspects of the theory. These are discussed below. The result provides independent justification of the plane-stress hypothesis adopted by Koiter [7-10] in his theory of shells. In turn, Koiter's hypothesis was motivated by John's [12] observation that plane stress obtains in the interior (i.e., away from edges) of a thin, three-dimensional elastic body in equilibrium with vanishing tractions at the lateral surfaces.

To prove the claim, let $U(\tilde{\mathbf{H}})$ and $\tilde{\mathbf{P}}(\tilde{\mathbf{H}})$ be the three-dimensional strain energy and stress at $\mathbf{x} \in R$ induced by the displacement field $\tilde{\mathbf{u}}(\mathbf{x})$. Using the decomposition (18), we define $G(\mathbf{d})=U(\nabla \hat{\mathbf{u}}+\mathbf{d} \otimes \mathbf{k})$, where $\mathbf{d}=\hat{\mathbf{u}}^{\prime}$. At a given material point the derivatives $\nabla \hat{\mathbf{u}}$ and $\hat{\mathbf{u}}^{\prime}$ may be specified independently. Thus, we fix the former and consider a one-parameter family $\mathbf{d}(u)$. The derivatives of $\sigma(u)=G(\mathbf{d}(u))$ are

$$
\dot{\sigma}=\tilde{\mathbf{P}} \mathbf{k} \cdot \dot{\mathbf{d}} \quad \text { and } \quad \ddot{\sigma}=\tilde{\mathbf{P}} \mathbf{k} \cdot \ddot{\mathbf{d}}+\dot{\mathbf{d}} \cdot(\tilde{\mathbf{P}} \mathbf{k})
$$

where

$$
(\tilde{\mathbf{P}} \mathbf{k})^{\cdot}=(\mathbf{C}[\dot{\mathbf{d}} \otimes \mathbf{k}]) \mathbf{k}
$$

Thus,

$$
G_{\mathbf{d}}=\tilde{\mathbf{P}} \mathbf{k}
$$

and

$$
\dot{\mathbf{d}} \cdot\left(R_{\mathbf{d d}}\right) \dot{\mathbf{d}}=\dot{\mathbf{d}} \otimes \mathbf{k} \cdot \mathbf{C}[\dot{\mathbf{d}} \otimes \mathbf{k}],
$$

which is positive-definite by virtue of (6). It follows that $\ddot{\sigma}>0$ on straight-line paths $\mathbf{d}(u)=(1-u) \mathbf{d}_{1}+$ $u \mathbf{d}_{2}$ with $u \in[0,1]$ and $\mathbf{d}_{1,2}$ fixed. These belong to the domain of $G(\cdot)$, the convex set generated by the linear space of 3 -vectors. Integrating with respect to $u$ then yields $\dot{\sigma}(u)>\dot{\sigma}(0)$ and $\sigma(1)-\sigma(0)>\dot{\sigma}(0)$, proving that $G(\mathbf{d})$ is a strictly convex function; i.e.,

$$
G\left(\mathbf{d}_{2}\right)-G\left(\mathbf{d}_{1}\right)>G_{\mathbf{d}}\left(\mathbf{d}_{1}\right) \cdot\left(\mathbf{d}_{2}-\mathbf{d}_{1}\right)
$$

for all unequal $\mathbf{d}_{1,2}$. Because such functions possess unique stationary points corresponding to absolute minima, it follows that

$$
U(\nabla \hat{\mathbf{u}}+\overline{\mathbf{d}}(\nabla \hat{\mathbf{u}}) \otimes \mathbf{k})<U(\nabla \hat{\mathbf{u}}+\mathbf{d} \otimes \mathbf{k})
$$

for all $\mathbf{d} \neq \overline{\mathbf{d}}(\nabla \hat{\mathbf{u}})$, where

$$
\hat{\mathbf{u}}^{\prime}=\overline{\mathbf{d}}(\nabla \hat{\mathbf{u}})
$$

is the unique solution to $\tilde{\mathbf{P}} \mathbf{k}=\mathbf{0}$. Therefore states of plane stress are energetically optimal when the two-dimensional displacement gradient $\nabla \hat{\mathbf{u}}$ is given. 
The three-dimensional strain energy is given by

$$
\mathcal{S}=\int_{R} U(\tilde{\mathbf{H}}(\mathbf{x})) d v=\int_{\Omega} W d a,
$$

where

$$
W=\int_{-h / 2}^{h / 2} U(\hat{\mathbf{H}}(\mathbf{r}, \varsigma)) d \varsigma
$$

is the areal strain-energy density. Leibniz' rule and Taylor's theorem imply that [4]

$$
W=h U_{0}+\frac{1}{24} h^{3} U_{0}^{\prime \prime}+o\left(h^{3}\right)
$$

where the subscript $(\cdot)_{0}$ identifies function values at $\varsigma=0$, as in (19) and (20). Thus,

$$
\begin{aligned}
U_{0} & =\frac{1}{2} \mathbf{H}_{0} \cdot \mathbf{C}_{0}\left[\mathbf{H}_{0}\right]=\frac{1}{2} \mathbf{P}_{0} \cdot \mathbf{H}_{0}, \\
U_{0}^{\prime} & =\mathbf{P}_{0} \cdot \mathbf{H}_{0}^{\prime} \text { and } U_{0}^{\prime \prime}=\mathbf{P}_{0}^{\prime} \cdot \mathbf{H}_{0}^{\prime}+\mathbf{P}_{0} \cdot \mathbf{H}_{0}^{\prime \prime},
\end{aligned}
$$

where $\mathbf{P}_{0}^{(n)}=\hat{\mathbf{P}}_{\mid \varsigma=0}^{(n)}$ with $\hat{\mathbf{P}}(\mathbf{r}, \varsigma)=\tilde{\mathbf{P}}(\mathbf{x})$ and, from (2),

$$
\mathbf{P}_{0}=\mathbf{C}_{0}\left[\mathbf{H}_{0}\right] \text { and } \quad \mathbf{P}_{0}^{\prime}=\mathbf{C}_{0}\left[\mathbf{H}_{0}^{\prime}\right]
$$

Further, it follows from (2) and (19) that

$$
\begin{aligned}
& \mathbf{P}_{0} \cdot \mathbf{H}_{0}=\mathbf{P}_{0} \mathbf{1} \cdot \nabla \mathbf{u}+\mathbf{a} \cdot \mathbf{P}_{0} \mathbf{k}, \quad \mathbf{P}_{0}^{\prime} \cdot \mathbf{H}_{0}^{\prime}=\mathbf{P}_{0}^{\prime} \mathbf{1} \cdot \nabla \mathbf{a}+\mathbf{b} \cdot \mathbf{P}_{0}^{\prime} \mathbf{k} \\
& \mathbf{P}_{0} \cdot \mathbf{H}_{0}^{\prime}=\mathbf{P}_{0} \mathbf{1} \cdot \nabla \mathbf{a}+\mathbf{b} \cdot \mathbf{P}_{0} \mathbf{k} \quad \text { and } \quad \mathbf{P}_{0} \cdot \mathbf{H}_{0}^{\prime \prime}=\mathbf{P}_{0} \mathbf{1} \cdot \nabla \mathbf{b}+\mathbf{c} \cdot \mathbf{P}_{0} \mathbf{k}
\end{aligned}
$$

The expression (31) for $W$ is not the complete strain energy for the order- $\varsigma^{3}$ ansatz defined by (21). However, our aim here is to derive an accurate order- $h^{3}$ expression for the energy rather than a complete expression for a given ansatz (cf. [13]).

Let $C^{*}$ be the line orthogonal to $\Omega$ and intersecting $\partial R$ at a point with position $\mathbf{r}$, and let $\partial R_{C}=$ $\partial \Omega \times C$, where $C$ is the collection of such lines, be the cylindrical generating surface of the platelike region $R$ obtained by translating the points of $\partial \Omega$ along their associated lines $C^{*}$. Let $s$ measure arclength on the curve $\partial \Omega$ with unit tangent $\boldsymbol{\tau}$ and rightward unit normal $\boldsymbol{\nu}=\boldsymbol{\tau} \times \mathbf{n}$.

Suppose, for the moment, that null tractions are assigned on the lateral surfaces of the plate. The assigned traction is then non-zero only on $\partial R_{C}$. The potential energy of the boundary tractions may thus be expanded to obtain

$$
\int_{\partial R_{t}} \tilde{\mathbf{t}} \cdot \tilde{\mathbf{u}} d a=\int_{\partial \Omega_{t}}\left(\mathbf{p}_{u} \cdot \mathbf{u}+\mathbf{p}_{a} \cdot \mathbf{a}+\mathbf{p}_{b} \cdot \mathbf{b}\right) d s+o\left(h^{3}\right)
$$

where $\partial \Omega_{t} \times C \subset \partial R_{C}$, and

$$
\mathbf{p}_{u}=h \mathbf{t}_{0}+\frac{1}{24} h^{3} \mathbf{t}_{0}^{\prime \prime}, \quad \mathbf{p}_{a}=\frac{1}{12} h^{3} \mathbf{t}_{0}^{\prime}, \quad \mathbf{p}_{b}=\frac{1}{24} h^{3} \mathbf{t}_{0},
$$

with $\mathbf{t}_{0}^{(n)}=\hat{\mathbf{t}}_{\mid \varsigma=0}^{(n)}$, where $\hat{\mathbf{t}}(\mathbf{r}, \varsigma)=\tilde{\mathbf{t}}(\mathbf{x})$ is the three-dimensional traction distribution on $\partial R_{t} \cap \partial R_{C}$. The complement of $\partial \Omega_{t}$ with respect to $\partial \Omega$ is $\partial \Omega_{u}$, where $\partial \Omega_{u} \times C=\partial R_{u} \subset \partial R_{C}$. Thus, position is assigned only on a part of $\partial R_{C}$, in accordance with the assignment of (null) tractions on the lateral surfaces. This furnishes

$$
E=\int_{\Omega}\left(h U_{0}+\frac{1}{24} h^{3} U_{0}^{\prime \prime}\right) d a-\int_{\partial \Omega_{t}}\left(\mathbf{p}_{u} \cdot \mathbf{u}+\mathbf{p}_{a} \cdot \mathbf{a}+\mathbf{p}_{b} \cdot \mathbf{b}\right) d s
$$




\subsection{Construction of the optimal plate energy}

Our objective is an expression for the order- $h^{3}$ approximation to the potential energy that is as accurate as possible by the standard of the three-dimensional theory. To this end we use the decomposition $\tilde{\mathbf{P}}=\tilde{\mathbf{P}} \mathbf{1}+\tilde{\mathbf{P}} \mathbf{k} \otimes \mathbf{k}$ and evaluate the exact equilibrium equation (1), expressed in the form

$$
\operatorname{div}(\tilde{\mathbf{P}} \mathbf{1})+\tilde{\mathbf{P}}^{\prime} \mathbf{k}=\mathbf{0}
$$

on the plane $\Omega$, obtaining

$$
\operatorname{div}\left(\mathbf{P}_{0} \mathbf{1}\right)+\mathbf{P}_{0}^{\prime} \mathbf{k}=\mathbf{0}
$$

Using (2) and (19), this is seen to furnish a relation among the fields $\mathbf{u}, \mathbf{a}$ and $\mathbf{b}$. Here it is imposed as a constraint in the approximate expression for the potential energy of the plate. Thus we use partial information about three-dimensional equilibria to generate the optimal order- $h^{3}$ potential energy functional for the midsurface deformation. In other words, although at this stage the mid-plane displacement field $\mathbf{u}(\mathbf{r})$ is unrestricted, the expression for the potential energy, to be derived, is not valid for arbitrary kinematically possible three-dimensional states. These states belong to a manifold of configurations, parametrized by $\mathbf{u}(\mathbf{r})$ and defined by equilibrium constraints on the directors. Stationarity of the energy then selects those midsurface displacement fields that approximate equilibria of the plate.

In addition to (39), Taylor expansions of the exact tractions $\tilde{\mathbf{t}}^{ \pm}= \pm \tilde{\mathbf{P}}^{ \pm} \mathbf{k}$ at the upper and lower lateral surfaces $\varsigma= \pm h / 2$, with unit normals $\mathbf{n}= \pm \mathbf{k}$, furnish

$$
\tilde{\mathbf{t}}^{+}+\tilde{\mathbf{t}}^{-}=h \mathbf{P}_{0}^{\prime} \mathbf{k}+O\left(h^{3}\right), \quad \tilde{\mathbf{t}}^{+}-\tilde{\mathbf{t}}^{-}=2 \mathbf{P}_{0} \mathbf{k}+O\left(h^{2}\right) .
$$

Therefore, if the lateral surfaces are traction free; i.e., if $\tilde{\mathbf{t}}^{ \pm}$vanish, then

$$
\mathbf{P}_{0} \mathbf{k}=O\left(h^{2}\right) \quad \text { and } \quad \mathbf{P}_{0}^{\prime} \mathbf{k}=O\left(h^{2}\right)
$$

Equations (39) and (41) are used to refine the expression for the order- $h^{3}$ plate energy.

Equation $(41)_{2}$ contrasts with the claim $\mathbf{P}_{0}^{\prime} \mathbf{k}=O(h)$ made in [13]. In that work no account is taken of restrictions imposed by the exact lateral traction data ${ }^{1}$. Evidently the motivation for the claim is to obtain a model, purportedly accurate to order $h^{3}$, that accounts for the effects of transverse shear while ensuring that the associated energy functional is strongly elliptic ${ }^{2}$. It may be concluded from the present considerations that this objective is achieved at the expense of accuracy, as judged by the equations and boundary data of the three-dimensional theory.

In view of (31)-(33), the complete order- $h^{3}$ expansion of the strain energy is

$$
W=\frac{1}{2} h\left(\mathbf{P}_{0} \mathbf{1} \cdot \nabla \mathbf{u}+\mathbf{a} \cdot \mathbf{P}_{0} \mathbf{k}\right)+\frac{1}{24} h^{3}\left(\mathbf{P}_{0}^{\prime} \mathbf{1} \cdot \nabla \mathbf{a}+\mathbf{b} \cdot \mathbf{P}_{0}^{\prime} \mathbf{k}+\mathbf{P}_{0} \mathbf{1} \cdot \nabla \mathbf{b}+\mathbf{c} \cdot \mathbf{P}_{0} \mathbf{k}\right)
$$

This expression is simplified by imposing the restrictions (39) and (41) associated with the exact threedimensional theory. Because these are necessary for energy minimizers in the exact theory, their imposition here yields the optimal order- $h^{3}$ truncation of the energy vis á vis three-dimensional elasticity theory. To this end, we use (39) to conclude that

$$
\mathbf{P}_{0} \mathbf{1} \cdot \nabla \mathbf{b}=\operatorname{div}\left[\left(\mathbf{P}_{0} \mathbf{1}\right)^{t} \mathbf{b}\right]-\mathbf{b} \cdot \operatorname{div}\left(\mathbf{P}_{0} \mathbf{1}\right)=\operatorname{div}\left[\left(\mathbf{P}_{0} \mathbf{1}\right)^{t} \mathbf{b}\right]+\mathbf{b} \cdot \mathbf{P}_{0}^{\prime} \mathbf{k}
$$


According to (41), the terms involving $\mathbf{P}_{0} \mathbf{k}$ and $\mathbf{P}_{0}^{\prime} \mathbf{k}$ make no contribution to the coefficient of $h^{3}$ in (42). Rather, they contribute at higher order in $h$, making them irrelevant to our development. To be precise, the stated accuracy is achieved by imposing

$$
\mathbf{P}_{0} \mathbf{k}=\mathbf{0} \quad \text { and } \quad \mathbf{P}_{0}^{\prime} \mathbf{k}=\mathbf{0}
$$

in the coefficient of $h^{3}$; the induced error in the potential energy is of order $h^{5}$, according to the threedimensional theory. The imposition of $(44)_{1,2}$ in the coefficient of $h^{3}$ is therefore consistent with our objective of deriving an optimal order- $h^{3}$ model. Using (2) and (19), these are found to yield

$$
\mathbf{A a}=-(\mathbf{C}[\nabla \mathbf{u}]) \mathbf{k} \quad \text { and } \quad \mathbf{A b}=-(\mathbf{C}[\nabla \mathbf{a}]) \mathbf{k},
$$

where $\mathbf{A}$ is the acoustic tensor defined by

$$
\mathbf{A v}=(\mathbf{C}[\mathbf{v} \otimes \mathbf{k}]) \mathbf{k}
$$

for any vector $\mathbf{v}$. In terms of components, $A_{i k}=C_{i 3 k 3}$. That this is positive definite follows from (6). Accordingly, $(45)_{1,2}$ yield $\mathbf{a}$ and $\mathbf{b}$ as functions of $\nabla \mathbf{u}$ and $\nabla \nabla \mathbf{u}$, respectively. We write

$$
\mathbf{a}=\overline{\mathbf{a}}(\nabla \mathbf{u}) \quad \text { and } \quad \mathbf{b}=\overline{\mathbf{b}}(\nabla \nabla \mathbf{u})
$$

where $\overline{\mathbf{b}}$ follows from $(45)_{2}$ in which $\nabla \mathbf{a}=\nabla \overline{\mathbf{a}}(\nabla \mathbf{u})$. Because the material is uniform, this depends only on $\nabla \nabla \mathbf{u}$.

This line of reasoning may not be used to suppress $\mathbf{P}_{0} \mathbf{k}$ in the coefficient of $h$ because $(41)_{1}$ implies that this term makes a net contribution to the energy at order $h^{3}$ and therefore that it is comparable to other terms that have been retained in (42). However, the coefficient of $h$ in the expression for the strain energy is the mid-plane value of the (positive-definite) three-dimensional strain energy. Applying the energetic argument of Section 2.1, we conclude that this term is optimized, for a given mid-plane displacement field $\mathbf{u}(\mathbf{r})$, by fixing the director field $\mathbf{a}(\mathbf{r})$ such that $\mathbf{P}_{0} \mathbf{k}=\mathbf{0}$. Accordingly, the restrictions (44) (and hence (45)) apply to all terms in the order- $h^{3}$ energy.

Taken together, our results imply that the optimal order- $h^{3}$ strain energy is given by

$$
\mathcal{S}=\int_{\Omega} \bar{W} d a+\frac{1}{24} h^{3} \int_{\partial \Omega} \mathbf{P}_{0} \mathbf{1} \boldsymbol{\nu} \cdot \mathbf{b} d s+o\left(h^{3}\right),
$$

where, as in $[4,5]$, Green's theorem has been used to transform the divergence in (43), $\boldsymbol{\nu}$ is the exterior unit normal to $\partial \Omega$, and

$$
\bar{W}=\frac{1}{2} h \mathbf{P}_{0} \mathbf{1} \cdot \nabla \mathbf{u}+\frac{1}{24} h^{3} \mathbf{P}_{0}^{\prime} \mathbf{1} \cdot \nabla \overline{\mathbf{a}}
$$

wherein (cf. (2) and (19))

$$
\mathbf{P}_{0}=\mathbf{C}[\nabla \mathbf{u}+\overline{\mathbf{a}}(\nabla \mathbf{u}) \otimes \mathbf{k}] \quad \text { and } \quad \mathbf{P}_{0}^{\prime}=\mathbf{C}[\nabla \overline{\mathbf{a}}(\nabla \mathbf{u})+\overline{\mathbf{b}}(\nabla \nabla \mathbf{u}) \otimes \mathbf{k}]
$$

It is noteworthy that the term $\mathbf{P}_{0}^{\prime} \mathbf{1} \cdot \nabla \overline{\mathbf{a}}$ in (49) and (50) 2 , obtained by imposing $(44)_{2}$, furnishes the minimum of the quadratic form $\mathbf{H}_{0}^{\prime} \cdot \mathbf{C}_{0}\left[\mathbf{H}_{0}^{\prime}\right]$ with respect to the director $\mathbf{b}$. The proof proceeds exactly as in (22)-(28), but with $G(\mathbf{d})$ replaced by $U\left(\nabla \hat{\mathbf{u}}^{\prime}+\mathbf{d}^{\prime} \otimes \mathbf{k}\right)$, where $\mathbf{d}^{\prime}=\hat{\mathbf{u}}^{\prime \prime}$.

Noting that $\mathbf{t}_{0}=\mathbf{P}_{0} \mathbf{1} \boldsymbol{\nu}$, the contribution to the boundary integral arising from $\partial \Omega_{t}$ is seen to balance the contribution $\mathbf{p}_{b}$ to the load potential (cf. (36)) in the expression (35) for the potential 
energy, leaving a residual integral over $\partial \Omega_{u}$ of $\mathbf{P}_{0} \mathbf{1} \boldsymbol{\nu} \cdot \mathbf{b}$. However, the latter is fixed by the data on $\partial \Omega_{u}$ and thus contributes only a disposable constant to the overall energy $[4,5]$. To see this we recall that $\tilde{\mathbf{u}}$ is assigned on $\partial R_{u}$. Consequently, its (tangential) derivatives with respect to $\varsigma$ are also assigned. This then fixes the values of $\mathbf{u}, \mathbf{a}$ and $\mathbf{b}$ on $\partial \Omega_{u}$. If the present model is to apply on the closure of $\Omega$, then the values of $\mathbf{a}$ and $\mathbf{b}$ obtained from (45) must agree with the data obtained from the assigned function $\tilde{\mathbf{u}}$ (cf. $(20)$ and $[4,5]$ ). This imposes a restriction on admissible data. Further, the decomposition [14]

$$
\nabla \mathbf{u}=\mathbf{u}_{s} \otimes \boldsymbol{\tau}+\mathbf{u}_{\nu} \otimes \boldsymbol{\nu}
$$

where $\mathbf{u}_{s}$ is the tangential (arclength) derivative on $\partial \Omega$, together with $(47)_{1}$, implies that the assignment of $\mathbf{u}$ and $\mathbf{a}$ on $\partial \Omega_{u}$ is tantamount to the assignment of $\mathbf{u}$ and $\mathbf{u}_{\nu}$ there. This in turn fixes the values of $\nabla \mathbf{u}$, and thus of $\mathbf{P}_{0} \mathbf{1} \boldsymbol{\nu}$, on $\partial \Omega_{u}$. With $\mathbf{b}$ fixed by the assigned $\tilde{\mathbf{u}}$, the residual integral over $\partial \Omega_{u}$ is then controlled by the data. It may therefore be suppressed, yielding the final expression

$$
\bar{E}=\int_{\Omega} \bar{W} d a-\int_{\partial \Omega_{t}}\left(\mathbf{p}_{u} \cdot \mathbf{u}+\mathbf{p}_{a} \cdot \overline{\mathbf{a}}\right) d s
$$

for the order- $h^{3}$ potential energy.

In the absence of distributed loads, correlation with the expression (10) for the plate energy is achieved via the identification

$$
F(\nabla \mathbf{u}, \nabla \nabla \mathbf{u})=\bar{W}
$$

and the boundary energies are correlated by using (45) $)_{1}$ and (51), yielding

$$
\mathbf{p}_{a} \cdot \overline{\mathbf{a}}=-\left\{\mathbf{p}_{a} \cdot \mathbf{A}^{-1}(\mathbf{C}[\mathbf{u} \otimes \boldsymbol{\tau}]) \mathbf{k}\right\}_{s}+\left(\mathbf{p}_{a}\right)_{s} \cdot \mathbf{A}^{-1}(\mathbf{C}[\mathbf{u} \otimes \boldsymbol{\tau}]) \mathbf{k}+\mathbf{p}_{a} \cdot \mathbf{A}^{-1}\left(\mathbf{C}\left[\mathbf{u} \otimes \boldsymbol{\tau}_{s}\right]\right) \mathbf{k}-\mathbf{p}_{a} \cdot \mathbf{A}^{-1}\left(\mathbf{C}\left[\mathbf{u}_{\nu} \otimes \boldsymbol{\tau}\right]\right) \mathbf{k}
$$

The variational derivative of the first term in this expression follows on replacing $\mathbf{u}$ therein by its variation $\dot{\mathbf{u}}$, say. This vanishes on $\partial \Omega_{u}$ and so the integral of the first term over $\partial \Omega_{t}$ is equivalent to its integral over $\partial \Omega$, which vanishes if the boundary is smooth; i.e., if $\boldsymbol{\tau}$ is continuous ${ }^{3}$. Accordingly, the first term in (54) has null variational derivative and therefore contributes only a disposable constant to the potential energy. The remaining terms are linear in $\mathbf{u}$ and $\mathbf{u}_{\nu}$. The Riesz representation theorem for linear scalar-valued functions then yields the existence of unique vectors $\mathbf{f}$ and $\mathbf{c}$ such that

$\mathbf{f} \cdot \mathbf{u}=\mathbf{p}_{u} \cdot \mathbf{u}+\left(\mathbf{p}_{a}\right)_{s} \cdot \mathbf{A}^{-1}(\mathbf{C}[\mathbf{u} \otimes \boldsymbol{\tau}]) \mathbf{k}+\mathbf{p}_{a} \cdot \mathbf{A}^{-1}\left(\mathbf{C}\left[\mathbf{u} \otimes \boldsymbol{\tau}_{s}\right]\right) \mathbf{k} \quad$ and $\quad \mathbf{c} \cdot \mathbf{u}_{\nu}=-\mathbf{p}_{a} \cdot \mathbf{A}^{-1}\left(\mathbf{C}\left[\mathbf{u}_{\nu} \otimes \boldsymbol{\tau}\right]\right) \mathbf{k}$

and with these results the functional $\bar{E}$ reduces to $E[\mathbf{u}]$ (cf. (10)).

The Euler equation associated with (10) and (53) is easily shown to predict that $\operatorname{div}\left(\mathbf{P}_{0} \mathbf{1}\right)=O\left(h^{2}\right)$. This is consistent with the exact equation (39), provided that $\mathbf{P}_{0}^{\prime} \mathbf{k}$ is likewise of order $h^{2}$, as was assumed in the foregoing derivation. Thus the present model is internally consistent and satisfies the lateral traction data of the three-dimensional theory with an error of order $h^{2}$, provided that eqs. (11)(13) deliver the mid-plane displacement field with the same error. If the plate is loaded by small tractions $\tilde{\mathbf{t}}^{ \pm}=O\left(h^{3}\right)$ at the lateral surfaces; i.e., if $\tilde{\mathbf{t}}^{ \pm}=h^{3} \mathbf{t}^{ \pm}+o\left(h^{3}\right)$, with $\mathbf{t}^{ \pm}$of order unity, then the foregoing model is augmented by a distributed-load potential $\mathbf{g} \cdot \mathbf{u}$ (cf. (10)), where

$$
\mathbf{g}=h^{3}\left(\mathbf{t}^{+}+\mathbf{t}^{-}\right)
$$


is the leading-order contribution to the net lateral traction. This is the scaling assumed in classical plate theory [15], and is accommodated without affecting the error in the lateral tractions.

The error may be reduced to order $h^{3}$, without affecting the model, by selecting the director $\mathbf{c}$ such that $\mathbf{P}_{0}^{\prime \prime} \mathbf{k}$ vanishes; i.e., by imposing $\mathbf{c}=\overline{\mathbf{c}}$, where (cf. (2) and $\left.(19)_{3}\right)$

$$
\mathbf{A} \overline{\mathbf{c}}=-(\mathbf{C}[\nabla \overline{\mathbf{b}}]) \mathbf{k}
$$

in which $\overline{\mathbf{b}}$ is computed a posteriori from solutions to eqs. (11)-(13). That this is possible follows from the fact that the director $\mathbf{c}$ plays no role in the model and is therefore at our disposal. Naturally, the claim about the error in representing the tractions presumes the same error in the mid-plane displacement field delivered by the model. In contrast, the system described in [13], based on the assumption $\mathbf{P}_{0}^{\prime} \mathbf{k}=O(h)$, carries a persistent error of order $h^{2}$ in the traction data.

\subsection{Energetic optimality of equilibria}

In view of (32)-(34), the strain-energy function defined by (49) and (50) may be expressed as

$$
\bar{W}=\frac{1}{2} h \overline{\mathbf{H}}_{0} \cdot \mathbf{C}\left[\overline{\mathbf{H}}_{0}\right]+\frac{1}{24} h^{3} \overline{\mathbf{H}}_{0}^{\prime} \cdot \mathbf{C}\left[\overline{\mathbf{H}}_{0}^{\prime}\right],
$$

where

$$
\overline{\mathbf{H}}_{0}=\nabla \mathbf{u}+\overline{\mathbf{a}} \otimes \mathbf{k} \quad \text { and } \quad \overline{\mathbf{H}}_{0}^{\prime}=\nabla \overline{\mathbf{a}}+\overline{\mathbf{b}} \otimes \mathbf{k},
$$

and the minor symmetries of $\mathbf{C}$ furnish

$$
\bar{W}=\frac{1}{2} h \mathbf{E} \cdot \mathbf{C}[\mathbf{E}]+\frac{1}{24} h^{3} \mathbf{E}^{\prime} \cdot \mathbf{C}\left[\mathbf{E}^{\prime}\right]
$$

where

$$
\mathbf{E}=\operatorname{Sym} \overline{\mathbf{H}}_{0} \quad \text { and } \quad \mathbf{E}^{\prime}=\operatorname{Sym} \overline{\mathbf{H}}_{0}^{\prime},
$$

yielding the strain energy as the sum of two positive-definite quadratic forms.

The potential energy $E[\mathbf{u}]$ is a quadratic functional. If $\mathbf{u}(\mathbf{r})=\mathbf{u}(\mathbf{r})+\dot{\mathbf{u}}(\mathbf{r})$ is a displacement field satisfying the data on $\partial \Omega_{u}$, it follows that

$$
E[\mathbf{u}]=E[\mathbf{u}]+\dot{E}[\mathbf{u}, \dot{\mathbf{u}}]+\frac{1}{2} \ddot{E}
$$

where $\dot{E}$ and $\ddot{E}$ are the induced first and second variations of the energy. Using (60), it is straightforward to obtain

$$
\ddot{E}=\int_{\Omega} \varphi d a
$$

where

$$
\varphi=h \dot{\mathbf{E}} \cdot \mathbf{C}[\dot{\mathbf{E}}]+\frac{1}{12} h^{3} \dot{\mathbf{E}}^{\prime} \cdot \mathbf{C}\left[\dot{\mathbf{E}}^{\prime}\right]
$$

and $\dot{\mathbf{E}}$ and $\dot{\mathbf{E}}^{\prime}$ are given by (59) and (61) in which $\mathbf{u}$ is replaced by $\dot{\mathbf{u}}$.

By definition, equilibria in this model are those displacements $\mathbf{u}(\mathbf{r})$ that nullify the first variation $\dot{E}[\mathbf{u}, \cdot]$, yielding

$$
E[\mathbf{u}]-E[\mathbf{u}]=\frac{1}{2} \int_{\Omega} \varphi d a
$$


Because $\varphi$ is non-negative, the integral can vanish only if $\varphi$ vanishes pointwise in $\Omega$, and as $\varphi$ is the sum of two positive-definite quadratic forms, this in turn requires that

$$
\dot{\mathbf{E}}=\mathbf{0} \quad \text { and } \quad \dot{\mathbf{E}}^{\prime}=\mathbf{0} \quad \text { in } \Omega \text {. }
$$

The analysis of these conditions is facilitated by decomposing the fields $\mathbf{u}, \mathbf{a}$ and $\mathbf{b}$ into parts parallel and orthogonal to $\Omega$. Thus,

$$
\mathbf{u}=\mathbf{v}+w \mathbf{k}, \quad \mathbf{a}=\boldsymbol{\alpha}+a \mathbf{k} \quad \text { and } \quad \mathbf{b}=\boldsymbol{\beta}+b \mathbf{k},
$$

where $\mathbf{v}=\mathbf{1} \mathbf{u}, \boldsymbol{\alpha}=\mathbf{1 a}$ and $\beta=\mathbf{1 b}$. Substituting the variations of these representations into (59) and (61), we derive

$$
\dot{\mathbf{E}}=\operatorname{Sym}[\nabla \dot{\mathbf{v}}+\mathbf{k} \otimes(\nabla \dot{w}+\dot{\boldsymbol{\alpha}})]+\dot{a} \mathbf{k} \otimes \mathbf{k} \quad \text { and } \quad \dot{\mathbf{E}}^{\prime}=\operatorname{Sym}[\nabla \dot{\boldsymbol{\alpha}}+\mathbf{k} \otimes(\nabla \dot{a}+\dot{\boldsymbol{\beta}})]+\dot{b} \mathbf{k} \otimes \mathbf{k}
$$

where $\operatorname{Sym}(\nabla \dot{\mathbf{v}})$ and $\operatorname{Sym}(\nabla \dot{\boldsymbol{\alpha}})$ are linear maps from the translation space $\Omega^{\prime}$ to itself. Accordingly, conditions (66) are satisfied if and only if

$$
\operatorname{Sym}(\nabla \dot{\mathbf{v}})=\mathbf{0}, \quad \dot{\boldsymbol{\alpha}}=-\nabla \dot{w}, \quad \nabla \nabla \dot{w}=\mathbf{0}, \quad \dot{\boldsymbol{\beta}}=\mathbf{0}, \quad \dot{a}=0 \quad \text { and } \quad \dot{b}=0 .
$$

It is easy to show that these restrictions are satisfied whenever the three-dimensional variation is a rigid-body displacement field. They also furnish trivial solutions to the constraints imposed by the variational derivatives of eqs. (44), upon which (64) is based.

In the three-dimensional parent problem, displacement is assigned on $\partial R_{u}$ and its variation vanishes there. As previously discussed, this implies that $\mathbf{u}$ and its normal derivative are assigned - and their variations vanish - on $\partial \Omega_{u}$. By using a decomposition like (51), this in turn is easily seen to imply that $\nabla \dot{w}=\mathbf{0}$ on $\partial \Omega_{u}$. Because $\nabla \dot{w}$ is uniform (cf. $\left.(69)_{3}\right)$, it then vanishes everywhere on the closure of $\Omega$, yielding a constant field $\dot{w}$ which vanishes by virtue of the data.

The restriction imposed by (69) ${ }_{1}$ yields $\nabla \dot{\mathbf{v}}=\omega \varepsilon$, where $\varepsilon=\varepsilon_{\alpha \beta} \mathbf{e}_{\alpha} \otimes \mathbf{e}_{\beta}$ is the skew two-dimensional permutation tensor $\left(\varepsilon_{12}=-\varepsilon_{21}=1, \varepsilon_{11}=\varepsilon_{22}=0\right)$ and $\omega(\mathbf{r}) \in \mathbb{R}$; the associated integrability condition is $\nabla \omega=\mathbf{0}$. Therefore, $\dot{\mathbf{v}}=(\omega \boldsymbol{\varepsilon}) \mathbf{r}+\mathbf{c}$ for some constants $\omega$ and $\mathbf{c}$. Differentiating this with respect to arclength on $\partial \Omega_{u}$, where $\dot{\mathbf{v}}$ vanishes identically, furnishes $\mathbf{0}=(\omega \boldsymbol{\varepsilon}) \boldsymbol{\tau}=\omega \boldsymbol{\nu}$ and hence $\omega=0$. Then $\mathbf{c}$ vanishes also, and we conclude that $\varphi$ vanishes in $\Omega$ if and only if $\dot{\mathbf{u}}$ vanishes identically; that is, if and only if the configuration of the plate is fixed. For variations that do not vanish identically, $\varphi$ is strictly positive in a set of non-zero area measure and its integral over $\Omega$ is then strictly positive. Equation (65) leads finally to the conclusion that an equilibrium displacement field is a minimizer of the energy; i.e., $E[\mathbf{u}] \geq E[\mathbf{u}]$, for all kinematically possible $\mathbf{u}$ not identically equal to $\mathbf{u}$. The minimum is strict if $\partial \Omega_{u}$ is non-empty and non-strict in the alternative case as rigid-body displacements are then admissible.

\section{Laminates}

To illustrate the extension of the foregoing ideas to laminates, we discuss in detail the case of a laminate composed of two laminae bonded together at the plane $\Omega$. We retain the convention that $\varsigma=0$ on $\Omega$. The upper and lower laminae occupy the regions $\Omega \times(0, \eta h]$ and $\Omega \times[-(1-\eta) h, 0)$, respectively, 
where $\eta \in(0,1)$ is the fraction of the total thickness $h$ apportioned to the upper lamina. We append subscripts $u$ and $l$ to variables defined in the upper and lower laminae, respectively.

The main adjustment of the foregoing development required to accommodate laminates is to the expression (30) for the strain energy, which is given here by

$$
W=\int_{0}^{\eta h} U_{u}\left(\hat{\mathbf{H}}_{u}(\mathbf{r}, \varsigma)\right) d \varsigma+\int_{-(1-\eta) h}^{0} U_{l}\left(\hat{\mathbf{H}}_{l}(\mathbf{r}, \varsigma)\right) d \varsigma .
$$

Leibniz' rule and a Taylor expansion, applied to both integrals, deliver

$$
\begin{aligned}
W= & h\left[\eta U_{u, 0}+(1-\eta) U_{l, 0}\right]+\frac{1}{2} h^{2}\left[\eta^{2} U_{u, 0}^{\prime}-(1-\eta)^{2} U_{l, 0}^{\prime}\right] \\
& +\frac{1}{6} h^{3}\left[\eta^{3} U_{u, 0}^{\prime \prime}+(1-\eta)^{3} U_{l, 0}^{\prime \prime}\right]+o\left(h^{3}\right)
\end{aligned}
$$

in lieu of (31), to which it reduces if the lamina properties are identical and $\eta=1 / 2$. This may be expressed in terms of the individual lamina deformation variables by using (32) and (34), which apply to both laminae at their interface $\Omega$.

The edge-load potential is treated in the same way, yielding

$$
\int_{\partial R_{t}} \tilde{\mathbf{t}} \cdot \tilde{\mathbf{u}} d a=\int_{\partial \Omega_{t}} P d s
$$

where

$$
P=\int_{0}^{\eta h} Q_{u} d \varsigma+\int_{-(1-\eta) h}^{0} Q_{l} d \varsigma \quad \text { and } \quad Q=\tilde{\mathbf{t}} \cdot \tilde{\mathbf{u}}
$$

Thus,

$$
\begin{aligned}
P= & h\left[\eta Q_{u, 0}+(1-\eta) Q_{l, 0}\right]+\frac{1}{2} h^{2}\left[\eta^{2} Q_{u, 0}^{\prime}-(1-\eta)^{2} Q_{l, 0}^{\prime}\right] \\
& +\frac{1}{6} h^{3}\left[\eta^{3} Q_{u, 0}^{\prime \prime}+(1-\eta)^{3} Q_{l, 0}^{\prime \prime}\right]+o\left(h^{3}\right),
\end{aligned}
$$

where

$$
Q_{0}=\mathbf{t}_{0} \cdot \mathbf{u}, \quad Q_{0}^{\prime}=\mathbf{t}_{0}^{\prime} \cdot \mathbf{u}+\mathbf{t}_{0} \cdot \mathbf{a} \quad \text { and } \quad Q_{0}^{\prime \prime}=\mathbf{t}_{0}^{\prime \prime} \cdot \mathbf{u}+2 \mathbf{t}_{0}^{\prime} \cdot \mathbf{a}+\mathbf{t}_{0} \cdot \mathbf{b},
$$

with subscripts ${ }_{u}$ or ${ }_{l}$ appended as appropriate. The displacement is continuous at the interface in the absence of delamination, implying that $\mathbf{u}_{u}=\mathbf{u}_{l}$; we continue to denote the common values of these functions by $\mathbf{u}(\mathbf{r})$.

We apply Green's theorem to the terms $\mathbf{P}_{0} \mathbf{1} \cdot \nabla \mathbf{a}$ and $\mathbf{P}_{0} \mathbf{1} \cdot \nabla \mathbf{b}$ appearing in $U_{0}^{\prime}$ and $U_{0}^{\prime \prime}$, respectively, as explained in Section 2.3. This generates integrals of $\mathbf{P}_{0} \mathbf{1} \boldsymbol{\nu} \cdot \mathbf{a}$ and $\mathbf{P}_{0} \mathbf{1} \boldsymbol{\nu} \cdot \mathbf{b}$ over $\partial \Omega$; their contributions from $\partial \Omega_{t}$ are balanced by the terms $\mathbf{t}_{0} \cdot \mathbf{a}$ and $\mathbf{t}_{0} \cdot \mathbf{b}$ appearing in $Q_{0}^{\prime}$ and $Q_{0}^{\prime \prime}$, respectively, leaving residual integrals over $\partial \Omega_{u}$ and integrals of $\mathbf{a} \cdot \operatorname{div}\left(\mathbf{P}_{0} \mathbf{1}\right)$ and $\mathbf{b} \cdot \operatorname{div}\left(\mathbf{P}_{0} \mathbf{1}\right)$ over $\Omega$. Combining these with (39), which holds in each lamina, we arrive at the lengthy preliminary expression

$$
\begin{aligned}
E= & \int_{\Omega}\left(h W_{1}+\frac{1}{2} h^{2} W_{2}+\frac{1}{6} h^{3} W_{3}\right) d a-\int_{\partial \Omega_{t}}\left(\mathbf{p}_{u} \cdot \mathbf{u}+\mathbf{p}_{a, u} \cdot \mathbf{a}_{u}+\mathbf{p}_{a, l} \cdot \mathbf{a}_{l}\right) d s \\
& +\frac{1}{2} h^{2} \int_{\partial \Omega_{u}}\left[\eta^{2} \mathbf{P}_{u, 0} \mathbf{1} \boldsymbol{\nu} \cdot \mathbf{a}_{u}-(1-\eta)^{2} \mathbf{P}_{l, 0} \mathbf{1} \boldsymbol{\nu} \cdot \mathbf{a}_{l}\right] d s \\
& +\frac{1}{6} h^{3} \int_{\partial \Omega_{u}}\left[\eta^{3} \mathbf{P}_{u, 0} \mathbf{1} \boldsymbol{\nu} \cdot \mathbf{b}_{u}+(1-\eta)^{3} \mathbf{P}_{l, 0} \mathbf{1} \boldsymbol{\nu} \cdot \mathbf{b}_{l}\right] d s
\end{aligned}
$$


for the order- $h^{3}$ potential energy, where

$$
\begin{aligned}
W_{1}= & \eta\left(\mathbf{P}_{u, 0} \mathbf{1} \cdot \nabla \mathbf{u}+\mathbf{a}_{u} \cdot \mathbf{P}_{0} \mathbf{k}\right)+(1-\eta)\left(\mathbf{P}_{0, l} \mathbf{1} \cdot \nabla \mathbf{u}+\mathbf{a}_{l} \cdot \mathbf{P}_{0} \mathbf{k}\right), \\
W_{2}= & \eta^{2}\left(\mathbf{a}_{u} \cdot \mathbf{P}_{u, 0}^{\prime} \mathbf{k}+\mathbf{b}_{u} \cdot \mathbf{P}_{0} \mathbf{k}\right)-(1-\eta)^{2}\left(\mathbf{a}_{l} \cdot \mathbf{P}_{l, 0}^{\prime} \mathbf{k}+\mathbf{b}_{l} \cdot \mathbf{P}_{0} \mathbf{k}\right), \\
\text { and } \quad W_{3}= & \eta^{3}\left[\mathbf{P}_{u, 0}^{\prime} \mathbf{1} \cdot \nabla \mathbf{a}_{u}+\mathbf{b}_{u} \cdot \mathbf{P}_{u, 0}^{\prime} \mathbf{k}+\left(\mathbf{b}_{u} \cdot \mathbf{P}_{u, 0}^{\prime} \mathbf{k}+\mathbf{c}_{u} \cdot \mathbf{P}_{0} \mathbf{k}\right)\right] \\
& +(1-\eta)^{3}\left[\mathbf{P}_{l, 0}^{\prime} \mathbf{1} \cdot \nabla \mathbf{a}_{l}+\mathbf{b}_{l} \cdot \mathbf{P}_{l, 0}^{\prime} \mathbf{k}+\left(\mathbf{b}_{l} \cdot \mathbf{P}_{l, 0}^{\prime} \mathbf{k}+\mathbf{c}_{l} \cdot \mathbf{P}_{0} \mathbf{k}\right)\right],
\end{aligned}
$$

with

$$
\begin{aligned}
\mathbf{p}_{u} & =h\left[\eta \mathbf{t}_{u, 0}+(1-\eta) \mathbf{t}_{l, 0}\right]+\frac{1}{2} h^{2}\left[\eta^{2} \mathbf{t}_{u, 0}^{\prime}-(1-\eta)^{2} \mathbf{t}_{l, 0}^{\prime}\right]+\frac{1}{6} h^{3}\left[\eta^{3} \mathbf{t}_{u, 0}^{\prime \prime}+(1-\eta)^{3} \mathbf{t}_{l, 0}^{\prime \prime}\right], \\
\mathbf{p}_{a, u} & =\frac{1}{3} h^{3} \eta^{3} \mathbf{t}_{u, 0}^{\prime}, \quad \mathbf{p}_{a, l}=\frac{1}{3} h^{3}(1-\eta)^{3} \mathbf{t}_{l, 0}^{\prime},
\end{aligned}
$$

and we have invoked the traction-continuity condition $\mathbf{P}_{u, 0} \mathbf{k}=\mathbf{P}_{l, 0} \mathbf{k}\left(\equiv \mathbf{P}_{0} \mathbf{k}\right)$ from the exact threedimensional theory.

As in the theory for laminae, this expression is dramatically simplified - and its accuracy enhanced by exploiting the lateral traction data in the three-dimensional theory. Thus, Taylor expansions of the tractions $\tilde{\mathbf{t}}^{+}=\tilde{\mathbf{P}}_{u}^{+} \mathbf{k}$ and $\tilde{\mathbf{t}}^{-}=-\tilde{\mathbf{P}}_{l}^{-} \mathbf{k}$, at the upper and lower lateral surfaces $\varsigma=\eta h$ and $\varsigma=-(1-\eta) h$ respectively, give

$\tilde{\mathbf{t}}^{+}=\mathbf{P}_{0} \mathbf{k}+\eta h \mathbf{P}_{u, 0}^{\prime} \mathbf{k}+\frac{1}{2} \eta^{2} h^{2} \mathbf{P}_{u, 0}^{\prime \prime} \mathbf{k}+O\left(h^{3}\right) \quad$ and $\quad \tilde{\mathbf{t}}^{-}=-\mathbf{P}_{0} \mathbf{k}+(1-\eta) h \mathbf{P}_{l, 0}^{\prime} \mathbf{k}-\frac{1}{2}(1-\eta)^{2} h^{2} \mathbf{P}_{l, 0}^{\prime \prime} \mathbf{k}+O\left(h^{3}\right)$.

We observe that if the lateral surfaces are unloaded, then the exact traction conditions $\tilde{\mathbf{t}}^{ \pm}=\mathbf{0}$ are satisfied with an error of order $h^{2}$ by taking ${ }^{4}$

$$
\mathbf{P}_{0} \mathbf{k}=\mathbf{0}, \quad \mathbf{P}_{u, 0}^{\prime} \mathbf{k}=\mathbf{0} \quad \text { and } \quad \mathbf{P}_{l, 0}^{\prime} \mathbf{k}=\mathbf{0} .
$$

Accordingly,

$$
\mathbf{a}_{u}=\overline{\mathbf{a}}_{u}(\nabla \mathbf{u}) \quad \text { and } \quad \mathbf{a}_{l}=\overline{\mathbf{a}}_{l}(\nabla \mathbf{u})
$$

where (cf. $\left.(45)_{1}\right)$

$$
\mathbf{A}_{u} \overline{\mathbf{a}}_{u}=-\left(\mathbf{C}_{u}[\nabla \mathbf{u}]\right) \mathbf{k} \quad \text { and } \quad \mathbf{A}_{l} \overline{\mathbf{a}}_{l}=-\left(\mathbf{C}_{l}[\nabla \mathbf{u}]\right) \mathbf{k}
$$

whereas

$$
\mathbf{b}_{u}=\overline{\mathbf{b}}_{u}(\nabla \nabla \mathbf{u}) \quad \text { and } \quad \mathbf{b}_{l}=\overline{\mathbf{b}}_{l}(\nabla \nabla \mathbf{u}),
$$

where (cf. $\left.(45)_{2}\right)$

$$
\mathbf{A}_{u} \overline{\mathbf{b}}_{u}=-\left(\mathbf{C}_{u}\left[\nabla \overline{\mathbf{a}}_{u}\right]\right) \mathbf{k} \quad \text { and } \quad \mathbf{A}_{l} \overline{\mathbf{b}}_{l}=-\left(\mathbf{C}_{l}\left[\nabla \overline{\mathbf{a}}_{l}\right]\right) \mathbf{k} .
$$

These conditions eliminate the coefficient of $h^{2}$, and some terms in the coefficients of $h$ and $h^{3}$, in the contribution to the potential energy from the integrals over $\Omega$. Moreover, the contributions from $\partial \Omega_{u}$ are then fixed by the data, as discussed in Section 2.3, and may therefore be eliminated. Thus we derive the order- $h^{3}$ potential energy (cf. (52))

$$
\bar{E}=\int_{\Omega} \bar{W} d a-\int_{\partial \Omega_{t}}\left(\mathbf{p}_{u} \cdot \mathbf{u}+\mathbf{p}_{a, u} \cdot \overline{\mathbf{a}}_{u}+\mathbf{p}_{a, l} \cdot \overline{\mathbf{a}}_{l}\right) d s
$$

where

$$
\bar{W}=h\left[\eta \mathbf{P}_{u, 0} \mathbf{1}+(1-\eta) \mathbf{P}_{0, l} \mathbf{1}\right] \cdot \nabla \mathbf{u}+\frac{1}{6} h^{3}\left[\eta^{3} \mathbf{P}_{u, 0}^{\prime} \mathbf{1} \cdot \nabla \overline{\mathbf{a}}_{u}+(1-\eta)^{3} \mathbf{P}_{l, 0}^{\prime} \mathbf{1} \cdot \nabla \overline{\mathbf{a}}_{l}\right],
$$


with

$$
\mathbf{P}_{0} \mathbf{1} \cdot \nabla \mathbf{u}=2 \overline{\mathbf{H}}_{0} \cdot \mathbf{C}\left[\overline{\mathbf{H}}_{0}\right] \quad \text { and } \quad \mathbf{P}_{0}^{\prime} \mathbf{1} \cdot \nabla \overline{\mathbf{a}}=\overline{\mathbf{H}}_{0}^{\prime} \cdot \mathbf{C}\left[\overline{\mathbf{H}}_{0}^{\prime}\right]
$$

in each lamina, where $\overline{\mathbf{H}}_{0}$ and $\overline{\mathbf{H}}_{0}^{\prime}$ are defined by (59) and subscripts ${ }_{u}$ or ${ }_{l}$ are appended as appropriate. It follows that the strain energy is the sum of four positive-definite quadratic forms, implying, as in the case of laminae, that equilibria furnish minima of the potential energy.

Expressions for the edge force $\mathbf{f}$ and couple $\mathbf{c}$ may be derived by using (85) together with obvious adjustments to (54) and (55), and the plate energy is again given by (10) in which $F=\bar{W}$ and $\bar{W}$ is given by (86).

\section{Notes}

1. In [13], restrictions imposed by (39) are adopted in the formulation of a model for plates, but the idea of using the exact three-dimensional equations to enhance the theory seems to be attributed, incorrectly, to [16]. The correct attribution is to $[4,5,17,18]$. In fact, the authors of [16] adopted the through-thickness average of (38), which has played a central role in the historical development of the subject (see [14], for example).

2. The work reported in [13] was evidently motivated by an observation in [4] to the effect that retention of all terms through order $h^{3}$ leads to a strain-energy function that violates the operative Legendre-Hadamard (semi-strict strong ellipticity) inequality, which is necessary for the existence of minimizers, implying that the associated energy functional does not admit of a minimum principle. To offset this undesirable feature, the condition $\mathbf{P}_{0}^{\prime} \mathbf{k}=\mathbf{0}$ imposed in $[4,5]$ and in the present paper is relaxed in [13], leading to an order- $h^{3}$ energy that satisfies the Legendre-Hadamard inequality. However, this is at odds with the estimate $(41)_{2}$ furnished by the exact theory; therefore, the restoration of ellipticity in [13] is achieved at the expense of accuracy. Moreover, the non-ellipticity reported in [4] is associated with the term $\mathbf{b} \cdot \operatorname{div}\left(\mathbf{P}_{0} \mathbf{1}\right)$, which we show here to be negligible in the order- $h^{3}$ theory.

With the benefit of hindsight we note that the considerations of [4] involve a plate possessing reflection symmetry with respect to its mid-plane. It follows that the in-plane and transverse displacements in that work decouple at leading order, and therefore that ellipticity is restored simply by retaining only the leading-order stretching and bending energies in the expression for the overall potential energy. This is explained in $[3,5]$.

In contrast, the present work yields an order- $h^{3}$ potential energy that is energetically optimal, of optimal accuracy vis á vis the exact theory, and applicable to materials exhibiting arbitrary symmetry. Further, its equilibria minimize the associated potential energy.

3. If $\partial \Omega$ is piecewise smooth then this term generates corner forces at the points where $\boldsymbol{\tau}$ is discontinuous. Such forces are well known in classical plate theory [15].

4. If conditions (80) were not imposed, then the expression (85) would contain terms involving $\mathbf{a} \cdot \mathbf{P}_{0}^{\prime} \mathbf{k}+\mathbf{b} \cdot \mathbf{P}_{0} \mathbf{k}$ at order $h^{2}$, and $\mathbf{b} \cdot \mathbf{P}_{0}^{\prime} \mathbf{k}+\mathbf{c} \cdot \mathbf{P}_{0} \mathbf{k}$ at order $h^{3}$ (the expressions in round parentheses in the second, third and fourth lines of (77)). These are not sign-definite. Their presence would imply that equilibria do not minimize the energy. Further, deformations could be constructed in such a way 
as to render the net contribution of these terms positive, implying that the associated expression for the energy is sub-optimal relative to that given here. The imposition of (80) achieves the threefold purpose of suppressing these indefinite terms, optimizing the remaining terms with respect to the directors a and $\mathbf{b}$ in each lamina (see the paragraph following (50)) and satisfying the lateral traction data through order $h$.

\section{Funding}

$O\left(h^{3}\right)$

\section{Conflict of interest}

$O\left(1 / h^{3}\right)$

\section{References}

1. G. Friesecke, S. Müller and R.D. James, 2002, Rigorous derivation of nonlinear plate theory and geometric rigidity. C.R. Acad. Sci. Paris, Ser I 334, 173-178.

2. P.G. Ciarlet, 2005, An introduction to differential geometry with applications to elasticity. J. Elasticity 78-79, 3-201.

3. R. Paroni, 2006, Theory of linearly elastic residually stressed plates. Math. Mech. Solids 11, $137-159$.

4. D.J. Steigmann, 2008, Two-dimensional models for the combined bending and stretching of plates and shells based on three-dimensional linear elasticity. Int. J. Engng. Sci. 46, 654-676.

5. D.J. Steigmann, 2009, Linear theory for the bending and extension of a thin, residually stressed, fiber-reinforced lamina. Int. J. Engng. Sci. 47, 1367-1378.

6. D.J. Steigmann, 2010, Elastic waves interacting with a thin, pre-stressed, fiber-reinforced surface film. Int. J. Engng. Sci. 48, 1604-1609.

7. W.T. Koiter, 1960, A consistent first approximation in the general theory of thin elastic shells, in: Proc. IUTAM Symposium on the Theory of Thin Elastic Shells, Delft (ed. W.T. Koiter). NorthHolland, Amsterdam, pp. 12-33.

8. W.T. Koiter, 1966, On the nonlinear theory of thin elastic shells. Proc. Knonklijke Nederlandse Akademie van Wetenschappen B69, 1-54.

9. W.T. Koiter, 1966, On the foundations of the linear theory of thin elastic shells, I and II. Proc. Knonklijke Nederlandse Akademie van Wetenschappen B78, 169-195.

10. W.T. Koiter, 1969, Foundations and basic equations of shell theory. A survey of recent progress, in: Theory of Thin Shells, Proc. IUTAM Symposium, Copenhagen (ed. F.I. Niordson). Springer, Berlin, pp. 93-115.

11. G. Fichera, 1972, Existence Theorems in Elasticity. In: Handbuch der Physik, vol. VIa/2 (C. 
Truesdell, ed.), pp. 347-389. Springer, Berlin.

12. F. John, 1965, Estimates for the derivatives of the stresses in a thin shell and interior shell equations. Comm. Pure \& Applied Math. 18, 235-267.

13. E. Pruchnicki, 2011, Two-dimensional model for the combined bending, stretching and transverse shearing of laminated plates derived from three-dimensional elasticity. Math. Mech. Solids 16, 304-316.

14. P.M. Naghdi, 1972, Theory of Shells and Plates. In: Handbuch der Physik, vol. VIa/2 (C. Truesdell, ed.), pp. 425-640. Springer, Berlin.

15. B.M. Fraeijs de Veubeke, 1979, A Course in Elasticity. Springer, New York.

16. A. DiCarlo, P. Podio-Guidugli and W.O. Williams, 2001, Shells with thickness distension. Int. J. Solids Structures 38, 1201-1225.

17. D.J. Steigmann, 2010, 'Applications of Polyconvexity and Strong Ellipticity to Nonlinear Elasticity and Elastic Plate Theory', in: CISM Course on Applications of Poly-, Quasi-, and Rank-One Convexity in Applied Mechanics, vol. 516 (J. Schröder and P. Neff, eds.). Springer, Wien and New York, pp. 265-299.

18. D.J. Steigmann, 2010, 'Recent developments in the theory of nonlinearly elastic plates and shells', in: Shell Structures, Theory and Applications, vol. 2 (W. Pietraszkiewicz and I. Krepa, eds.). Proc. 9th SSTA Conf. (Gdansk-Jurata, Poland, Oct. 14-16, 2009). CRC Press, Boca Raton, pp. 19-23. 